\title{
La subjectivité du sujet lecteur de poésie au lycée : un défi (possible ?) aux enseignants
}

\section{Cynthia Agra de Brito Neves}

\section{Q OpenEdition}

\section{Journals}

Édition électronique

URL : http://journals.openedition.org/recherchestravaux/653

DOI : 10.4000/recherchestravaux.653

ISSN : 1969-6434

Éditeur

UGA Éditions/Université Grenoble Alpes

\section{Édition imprimée}

Date de publication : 31 décembre 2013

Pagination : 101-115

ISBN : 978-2-84310-267-7

ISSN : 0151-1874

Référence électronique

Cynthia Agra de Brito Neves, «La subjectivité du sujet lecteur de poésie au lycée : un défi (possible ?) aux enseignants », Recherches \& Travaux [En ligne], 83 | 2013, mis en ligne le 01 juillet 2015, consulté le 08 septembre 2020. URL : http://journals.openedition.org/recherchestravaux/653 ; DOI : https:// doi.org/10.4000/recherchestravaux.653 


\section{La subjectivité du sujet lecteur de poésie au lycée : un défi (possible?) aux enseignants}

Les poésies à l'école peuvent encore donner l'occasion aux poésies et aux élèves de (se) lire, de (se) dire, de (s’)écrire. Les poésies se font chaque jour dans la lecture de tous, dans la parole de chacun, car aucun ne peut éviter «la rime et la vie ${ }^{2}$ ».

Ce travail fait partie de notre thèse de doctorat en cours d'élaboration, dans laquelle nous cherchons à étudier la façon dont les "poésies» ou "genres poétiques» - toujours au pluriel - circulent dans les classes de lycées brésiliens et français. Ainsi, il s'agit d'une étude comparative qui a pour objet l'enseignement et les voies d'apprentissage de la littérature à partir de l'observation des travaux de professeurs brésiliens et français sur des textes poétiques - traduits ou non et des réactions des élèves à ces textes dans les dynamiques de classe. Nous y comparons également les programmes nationaux, le matériel didactique, et l'Examen national de l'enseignement secondaire (Exame Nacional de Ensino Médio), l'ENEM brésilien, avec le baccalauréat français.

L'ENEM est une épreuve écrite que les élèves brésiliens passent à la fin de la troisième année du lycée. Cet examen national a pour objectif d'évaluer les compétences en lecture et interprétation de divers genres textuels, textes de langage verbal (énoncés littéraires et informatifs) et visuel (photos, cartes, peintures, graphiques, etc.) à travers des questionnaires à choix multiples. Il s'enquiert aussi de l'aptitude de l'élève à argumenter, à résoudre des problèmes pratiques de la vie quotidienne, à élaborer des propositions d'interventions réelles et à présenter des idées bien structurées au cours de la rédaction d'une

I. Doctorante à l'UNICAMP (bénéficiaire d'une bourse de la CAPES au Brésil) et au CEDILIT, Centre de recherche en didactique de la littérature.

2. M.-C. et S. Martin, Les Poésies, l'école, Paris, Presses universitaires de France, 1997, p. 138. 
dissertation. La réussite de l'élève à cette épreuve fait partie du processus qui permet son entrée à l'université, ses notes étant enregistrées et additionnées à celles du vestibular (l'épreuve d'entrée spécifique à chaque université). En ce sens, l'ENEM est comparable au baccalauréat.

Notre intérêt pour la pédagogie française est dû à son influence historique sur le système éducatif brésilien, principalement dans le domaine de l'enseignement des langues et de la littérature. La réalisation de ce travail a été possible en $20 I I$ grâce à l'opportunité de participer au CEDILIT (Centre de recherche en didactique de la littérature, dirigé par le professeur Jean-François Massol, de l'université Stendhal - Grenoble 3). En plus des réunions et discussions de ce groupe, nous avons suivi trente heures de cours de langue et littérature française en classes de secondes générales, de premières (économique et sociale, et littéraire) et de terminale littéraire dans deux lycées de Grenoble : le lycée Champollion (avec l'enseignante S. B.) en centre-ville, et le lycée Marie-Curie (avec l'enseignante V. G.) à Échirolles. Au Brésil, tout au long de l'année 2010 et au début de 20I2, nous avons également passé 30 heures dans des classes de langue et littérature portugaise et brésilienne équivalentes aux seconde, première et terminale, dans deux écoles de province de Sao Paulo : à Anglo-Taquaral de Campinas (avec l'enseignant F. M.), et au Colégio Objetivo d'Indaiatuba (avec l'enseignant S. P.). En tant que chercheuse-observatrice, nous avons participé, durant ces périodes et au sein de ces institutions, aux activités ayant trait aux poésies et, dans une perspective qualitative et subjective, nous avons recueilli des données dans un journal de bord qui constituera un des objets d'analyse de notre thèse.

Dans ce travail spécialement élaboré pour ce colloque «Le sujet lecteurscripteur de l'école à l'université : postures et outils pour des lecteurs divers et singuliers", nous anticipons quelques-unes de nos observations et critiques qui méritent ici un intérêt particulier, puisqu' elles ont trait à la réalisation de lectures et écritures subjectives de poésies dans les contextes scolaires français et brésilien, parfois de façon diamétralement opposées, et d'autres fois similaires.

\section{Comment les poésies (sur)vivent Entre les murs ${ }^{3}$ de l'institution scolaire : l'importance accordée à l'analyse formelle et la recherche des figures de style}

Au sein d'un espace institutionnel aussi bureaucratique, puissant et contrôleur de discours que l'entendait Michel Foucault ${ }^{4}$, il est difficile d'imaginer des

3. Nous faisons ici référence au film Entre les murs, du cinéaste français L. Cantet, Palme d'or au festival de Cannes en 2008. Il montre la dynamique d'une salle de classe en France et les conflits auxquels le professeur de français, F. Marin, est confronté au long de l'année scolaire. 4. M. Foucault, L'Ordre du discours, Paris, Gallimard, I97I. 
activités qui renvoient aux joies de la littératures ou au "plaisir du texte ${ }^{6}$ ", en particulier lorsqu'il s'agit de lectures poétiques. Dans l'univers de Roland Barthes, le texte est un discours qui attend d'être lu, qui cherche à séduire son lecteur, à le provoquer et à l'emporter. Se construit alors un espace de jouissance, un jeu dialectique qui fait de l'écriture la science des jouissances du langage, son kamasutra. Lorsqu'il lit, penché sur une matérialité pleine de ruptures et de collisions - la langue, son lexique, sa métrique, sa prosodie - le lecteur vit l'instant insoutenable où il peut atteindre la jouissance de la cohabitation des langues. Cette jouissance, c'est le plaisir du texte.

Or, ce sont justement les lectures poétiques qui sont, souvent, et de différentes façons, déviées voire niées par l'institution scolaire. Les professeurs dont nous avons suivi les classes semblent en avoir conscience et essaient, dans une certaine mesure, d'aller à contre-sens de cette logique. Cependant, ils en dépendent et soumettent leurs méthodes d'enseignement de la littérature aux diktats du baccalauréat et de l'ENEM. D'une certaine façon, ce sont les examens de fin de cycle secondaire qui régissent l'enseignement et donc l'apprentissage de la langue et de la littérature des deux pays en question.

En observant le déroulement de "séquences didactiques (SD) poétiques" en France et au Brésil, nous avons remarqué une prise en compte constante de l'examen national, aussi bien à travers le choix des professeurs d'enseigner tel ou tel poète, que l'intérêt manifeste des élèves pour le sujet traité en classe. En France, l'élève apprend à lire à voix haute en respectant les liaisons et les enjambements, il est entraîné à écrire des sous-parties pour produire un commentaire littéraire - tout cela dans la perspective du baccalauréat, comme nous le verrons plus loin. Au lycée brésilien, on ne relève pas de pratique de la lecture poétique à voix haute, puisque l'ENEM est une épreuve entièrement écrite. En revanche, on y enseigne, de façon insistante et depuis l'équivalent de la classe de seconde, les règles de la métrique, la scansion, les rimes, c'està-dire les aspects formels du texte poétique dont la connaissance est exigée lors de l'examen national.

En France, nous avons suivi un module entier de travail sur des poésies, du Symbolisme au Surréalisme, dans des classes de seconde générale du lycée Champollion. L'objectif principal de cette SD, a précisé Mme S. B., était la lecture complète du recueil des Fleurs du mal de Charles Baudelaire ${ }^{7}$. Les élèves devaient lire l'œuvre poétique à la maison et, en classe, l'enseignante reprenait la

5. G. Snyders, Alunos felizes: reflexão sobre a alegria na escola a partir de textos literários, trad. C. A. P. da Silva, Rio de Janeiro, Paz e Terra, I993.

6. R. Barthes, Le Plaisir du texte, Paris, Seuil, I973.

7. C. Baudelaire, Les Fleurs du Mal, Paris, Hachette Éducation, coll. «Bibliolycée», 2002. 
lecture des poèmes qu'elle avait sélectionnés. Après la lecture de chaque poème, la professeure choisissait, dans leur manuel, des questions d'interprétation du texte, les élèves répondaient, puis on passait à la correction de l'exercice.

L'un des premiers poèmes étudiés fut "Élévation». Peu après sa lecture, Mme S. B. a demandé si quelqu'un avait des questions de vocabulaire. Elle a ensuite expliqué ce qu'est "Le spleen et l'idéal » et s'est ensuite lancée dans une série de questions ouvertes à la classe, en demandant par exemple : "Quelle est la forme du poème?", "Combien y a-t-il de syllabes?», "Quelle est la rime?», "Quels sont les champs lexicaux?». Ce n'est qu’à la fin qu'elle s'est inquiétée de la polysémie du titre et qu'elle a écrit au tableau : «Élévation physique; élévation spirituelle/mentale; sens religieux = l'élévation de l'hostie» et a demandé aux élèves de relire le poème en considérant ces sens.

Pendant un autre cours, après la lecture de ce même poème, l'enseignante a montré l'anaphore «Par-delà» des vers 3 et 4, attiré l'attention sur l'impératif du vers 9 : «Envole-toi», expliqué l'idée métonymique du vers 15 «Heureux [...] aile vigoureuse = métonymie de l'oiseau / de l'ange», puis a alors parlé de la transcendance évoquée dans ce vers. Elle a exploré la question de la dualité pour expliquer l'antithèse et le paradoxe et, enfin, a demandé aux élèves d'identifier dans le poème des exemples d'enjambements.

Ainsi, les questions posées par Mme S. B. témoignent d'une préoccupation constante pour les aspects formels du poème et les figures de style. L'idée d'élévation comme libération, la transcendance spirituelle, la fuite du monde réel, le nirvana... tous ces aspects thématiques liés à l' "Élévation" sont interprétés et analysés dans la perspective d'une explication a priori formelle du poème.

La critique de Michèle Petit à l'égard de ce type d'enseignement est assez pertinente :

Et l'on a privilégié une conception instrumentale, formaliste, entièrement du côté de la maîtrise du texte - inspirée de la linguistique structurale et de la sémiotique, puis d'une néo-rhétorique [...]. Le «texte» littéraire est devenu une machine qui fonctionne - le lecteur étant supposé acquérir le savoir-faire technique, les outils à même de le faire fonctionner - puis une "forme de discours" parmi d'autres. [... plus] l'école s'ouvrait à des catégories sociales éloignées de la culture lettrée, plus s'y imposaient un jargon et des techniques d'une extrême sophistication, empruntés à la critique textuelle, aux théories de l'énonciation, à la rhétorique ${ }^{8}$.

Les jargons et le technicisme n'apprennent ni à lire ni à écrire, et encore moins - ajouterions-nous - à éveiller la subjectivité de l'élève comme sujet lecteur de poésies.

8. M. Petit, Éloge de la lecture : la construction de soi, Paris, Belin, 2002, p. I27-I28. 
Au Brésil, la didactique est comparable, et les manuels de langue et littérature portugaises au lycée proposent des exercices qui, comme l'observe Joaquim Brasil Fontes ${ }^{9}$, obligent l'élève à identifier les figures de style dans des fragments de textes, notamment de poésies, dans le but d'apprendre à reconnaitre les ressources stylistiques qui caractériseraient tel ou tel auteur, telle ou telle école littéraire. Il ajoute que la théorie des figures de style apparaît peu après les questions de grammaire de base et avant l'introduction aux concepts généraux de littérature.

Non seulement le manuel survalorise les «figures cachées » dans les discours, mais la stratégie même de l'enseignant vise l'identification et la classification des figures ou des fonctions du langage par exemple; nous l'avons observé dans les classes en France et au Brésil. Dans ces deux contextes scolaires, le plaisir des lectures poétiques, le goût des poésies ainsi que l'apprentissage d'une lecture, selon les mots de Michèle Petit "pour se découvrir ou se construire, pour élaborer son intériorité, sa subjectivité ${ }^{10}$ ", ne semblent occuper que le deuxième plan.

Ainsi, le sujet lecteur-scripteur cherche presque toujours à échapper à cette "prison formelle» - et, par extension, institutionnelle - en déviant l'attention de la classe vers ce qui l'intéresse de façon subjective. Nous relevons, dans ce travail, certains moments que nous avons vécus en classe, quand les élèves : I) ont montré de la curiosité pour la vie du poète (Baudelaire en France, Pessoa au Brésil) ; 2) ont tissé des interprétations personnelles et créatives d'un poème de Rimbaud ; 3) ont manifesté de l'émotion pendant leur lecture de poésies en les projetant dans leur vie privée.

\section{L'intérêt des élèves pour la personne de l'auteur}

Au lycée Champollion, Mme S. B. a intitulé sa séquence : «Le Symbolisme dans la poésie de Baudelaire; le spleen et l'idéal ». L'enseignante présente le poète, mais parle d'abord du mouvement littéraire dans lequel il s'inscrit. Elle souligne les caractéristiques du symbolisme en écrivant au tableau : "le contexte», «les grands principes", "les formes et genres", «les thèmes dominants", «les auteurs et les œuvres ". Elle donne ensuite le nom des "poètes maudits": «Baudelaire, Les Fleurs du mal (1857); Rimbaud, Illumination (I886); Verlaine,

9. J. B. Fontes, As obrigatórias metáforas: apontamentos sobre literatura e ensino, São Paulo, Iluminuras Ltda., 1999.

Io. M. Petit, Éloge de la lecture, ouvr. cité, p. 7. 
Poèmes saturniens (I866)». L'étape suivante consiste à livrer la biographie de Baudelaire, ce qui intéresse beaucoup les élèves.

La classe note ce que la professeure écrit au tableau tout en posant des questions d'intérêt personnel sur Baudelaire. Elle veut des détails, savoir par exemple pourquoi le poète s'était disputé avec son beau-père, s'il avait toujours été riche, s'il n'avait vécu que de l'héritage de son père, s'il s'était marié - puisqu'il était «bohémien» - quelles drogues il prenait, si l'absinthe était une boisson dont la teneur hallucinogène venait d'une fleur, comme l'opium. Enfin, la curiosité à propos de la vie et des drogues anime toute la salle. Le fait d'avoir été un poète outrageant la morale publique, censuré pour cela, et ses pièces ayant été condamnées, intéresse aussi les élèves qui veulent savoir s'il a été en prison, s'il a eu des relations homosexuelles - comme il traite du lesbianisme dans certains poèmes $-s^{\prime}$ il fréquentait des prostituées et ce que sa femme faisait quand elle l'apprenait, etc. On doit l'admettre, cette curiosité finit par détourner les élèves de tout intérêt pour la poésie baudelairienne.

Au Brésil, nous avons vécu une réalité similaire dans les classes équivalentes à la première et à la terminale ${ }^{\mathrm{II}}$ du Colégio Anglo de Campinas. D'abord, M. F. M. annonce qu'il va parler du plus important poète de langue portugaise $\mathrm{du} \mathrm{Xx}^{\mathrm{e}}$ siècle, celui qui dispute la première place à Camóes ( $\mathrm{xvI}^{\mathrm{e}}$ siècle); il plaisante en disant que «ce type est trop cool» puis révèle qu'il s'agit de Fernando Pessoa. Les élèves écoutent, attentifs et curieux. M. F. M. met en avant des faits de la vie privée du poète portugais : il avait peu d'amis, il n'a publié qu'un livre de son vivant (Message, 1934), ses autres poèmes ont été trouvés dans des coffres et aujourd'hui encore, ils intéressent par le génie de l'hétéronymie créée par le poète.

Avant d'expliquer les hétéronymes, le professeur est déjà surpris par les questions des élèves qui voudraient savoir si Pessoa s'est marié, s'il était homosexuel, s'il n'a écrit que de la poésie, la raison de sa mort, etc. Quand il précise que le poète est mort d'une cirrhose du foie, M. F. M. laisse les élèves faire des commentaires du type : «Encore un poète saoul!», renforçant ainsi le cliché qui associe poésie et vie de bohème. L'enseignant commence alors à expliquer en détails les hétéronymes créés par Pessoa, et écrit au tableau : «La création principale de Fernando Pessoa est ses hétéronymes (différents noms), personnalités poétiques indépendantes avec leurs propres biographie, poèmes

\footnotetext{
II. Les écoles brésiliennes dans lesquelles nous avons fait ces travaux d'observation cherchent à enseigner tout le contenu du programme de lycée durant les deux premières années et réservent la troisième à une révision générale. L'idée est d'utiliser cette dernière année pour entraîner les élèves aux épreuves de l'ENEM et du vestibular. La note obtenue à l'examen du vestibular est ajoutée à celle de l'ENEM, et c'est ce résultat qui permet (ou non) l'entrée de l'élève dans l'enseignement supérieur. Au Brésil, une grande partie de l'enseignement privé organise son programme de cette façon, privilégiant la révision.
} 
et style dans lesquels se fragmente le poète.» Il explique la différence entre pseudonyme et hétéronyme, et donne les trois principaux hétéronymes du poète portugais : Alberto Caeiro, Ricardo Reis et Alvaro de Campos.

Alors qu'il écrit au tableau les caractéristiques de chaque hétéronyme, il est interrompu par les commentaires des élèves, toujours très intéressés par la vie de chacun d'eux et par celle de Pessoa : "Ce Fernando Pessoa était fou?», "C'était un fake?», "Il était bipolaire?», "C'est un crime d'inventer des personnes qui n'existent pas, c'est de l'usage d'identité fictive!», «Personne ne l'a mis en prison? », «Il devait fumer à mort, c'est sûr!», «Il se droguait, ce pauvre vieux...", "Moi, je crois que même Fernando Pessoa était un hétéronyme!», «Tu parles, il était schizophrène, oui!».

Les réactions des élèves tout au long du cours indiquent une indignation d'ordre subjectif. L'enseignant cherche à faire des associations avec les films Matrix $^{12}$ et Un homme d'exception ${ }^{13}$ en expliquant que nous aussi sommes des êtres multiples, que nous nous dédoublons en plusieurs versions, que nous sommes aussi divers personnages réels ou virtuels, comme sur les réseaux sociaux. Rien ne calme l'euphorie des élèves, impressionnés par l'hétéronymie de Pessoa. Ils montrent peu d'intérêt pour les poésies lues et présentées par le professeur ("Autopsychographie» de Pessoa «Lui-même»; "Le Gardien de troupeau" d'Alberto Caeiro; "Viens t'asseoir avec moi, Lydia, au bord de la rivière" de Ricardo Reis; "Lisbon Revisited" d'Alvaro de Campos $\left.{ }^{\mathrm{I}}\right)$, et ne veulent connaitre que la vie de chaque poète créé par le plus grand.

Avant même que les élèves ne s'approprient les textes poétiques, ils s'attachent à la vie de leurs auteurs et, en ce sens, nous pouvons dire qu'ils réalisent une lecture subjective. Au Brésil comme en France, les enseignants essaient de minimiser les aspects biographiques et de valoriser les textes poétiques en classe, mais ils ne parviennent pas à faire avancer le cours comme ils le souhaiteraient car ils sont sans cesse interrompus par les questions et commentaires de leurs élèves qui détournent l'objectif de la classe vers la vie privée des poètes.

\section{Les fantômes du bac et de l'ENEM}

Quand le mouvement se fait dans le sens inverse, c'est le professeur qui est frustré. C'est ce qui se passe quand Mme S. B. termine la biographie de Baudelaire et commence à travailler sur les lectures des Fleurs $d u$ Mal, consacrant

I2. Matrix, production cinématographique de 1999 dirigée par les frères Wachowski.

I3. Un homme d'exception (en anglais, Beautiful Mind), drame biographique de 200 s sur la vie du mathématicien John Forbes Nash, dirigé par Ron Howard.

I4. F. Pessoa, Obra Poética, Rio de Janeiro, Nova Aguilar SA, 1994. 
plusieurs cours à la lecture de "Spleen", "Parfum exotique», "L'Albatros», «Les Bijoux» et "Femmes damnées». Ces poèmes sont lus à voix haute par l'enseignante à cause de leur longueur alors qu'habituellement, d'après ce que nous avons remarqué dans les deux lycées français, ce sont les élèves qui lisent les poèmes.

Mme S. B. attire l'attention sur la scène de lesbianisme et d'inceste dans «Femmes damnées"; toutefois, à notre surprise et à la sienne, le thème provocant n'éveille pas l'intérêt des élèves de seconde générale. Ils se soucient plutôt d'apprendre rapidement à faire le commentaire composé - objectif final des lectures de Baudelaire - puisque c'est ce qui leur sera demandé lors du devoir surveillé de la semaine suivante. La professeure s'attendait à une réaction émotionnelle de la part des adolescents lors de cette lecture érotique, et elle est déçue en voyant qu'ils donnent la priorité à l'aspect formel : ils se privent du plaisir du texte et de la lecture subjective en donnant une plus grande importance à l'évaluation institutionnelle.

Nous pouvons dire que la lecture des Fleurs du Mal au lycée Champollion a presque toujours été dirigée et, en ce sens, réduite à l'apprentissage d'un type de texte spécifique : le commentaire composé. Ce type de rédaction littéraire n'est pas enseigné dans les écoles brésiliennes, mais on lui accorde une grande importance sur le territoire français. L'ENEM brésilien en revanche, est une épreuve à choix multiples qui porte aussi sur l'écriture d'un texte, dans notre cas une dissertation - à caractère non littéraire -, mais dont le sujet est un thème d'actualité à propos duquel l'élève doit argumenter de façon cohérente et objective. L'épreuve exige également que l'élève termine son texte en proposant des solutions au problème posé et prenne en considération les droits de l'homme. Les enseignants brésiliens passent l'année scolaire à essayer de deviner le thème qui sera choisi pour l'ENEM et dirigent la pratique de production de texte de façon à entraîner leurs étudiants à élaborer une dissertation.

Soulignons une autre comparaison. Dans les lycées français, les professeurs demandent aux élèves de lire à voix haute les poésies comme entraînement à la lecture expressive pour l'examen oral du bac - évaluation réalisée en fin de classe de première. Au Brésil, la lecture de poésies à voix haute n’est pas pratiquée par les élèves de lycée, très probablement parce que l'ENEM ne propose pas d'épreuve orale. En France, le rituel demande que l'élève se lève pour lire à voix haute le poème choisi et fasse attention à sa posture corporelle, à sa diction, à son intonation, au rythme des vers. Au Brésil, nous l'avons observé dans les classes, ce rituel est différent. C'est le professeur qui fait la lecture, souvent en la théâtralisant comme aime le faire M. S. P., ou en déclamant les vers appris par cœur comme le fait parfois M. F. M. 
La lecture faite par les enseignants est une stratégie pour faciliter la compréhension des élèves. En lisant à voix haute, MM. S. P. et F. M. donnent un rythme au poème, gesticulent, lisent avec le corps, à travers le corps, in-corpo-rant les poésies avec "les techniques du corps", dirait Marcel Mauss ${ }^{15}$. Selon les mots de Marie-Claire et Serge Martin :

On recherche[...] une justesse, non au sens où une diction viendrait subsumer toutes les autres, mais parce qu'à un moment donné, dans une lecture donnée, le poème dit aurait créé un rapport entre ses différents protagonistes, entre toutes les composantes de sa diction pour laisser agir à plein la signifiance. Il s'agit de [...] dire avec son corps, le corps disant devenant le poème dans ce mouvement d'incorporation. Il faut chercher ce lieu de la voix car changer de diction peut être changer de poème ${ }^{16}$.

Les élèves apprécient ce théâtre, ils sont impressionnés par la mémoire de M. F. M. qui déclame par cœur les vers de Fernando Pessoa et de Camões, et ils demandent à M. S. P. de lire le poème à haute voix car ils savent qu'il aime ça et qu'il est aussi poète - il partage des poèmes écrits de sa plume sur Facebook. Les élèves adorent cette affinité des enseignants avec les poésies et quand ils osent à leur tour griffonner quelques vers, ils demandent à leurs maitres de les lire et de les commenter - mais cette fois en secret, loin de la salle de classe, comme nous l'ont rapporté les professeurs.

\section{La lecture irrévérencieuse et créative de $\mathrm{C}$.}

Il est intéressant de remarquer aussi les différences de goûts des élèves, selon leur sexe. La relation des garçons et des filles à la lecture - et par extension, ajouterions-nous, au poétique - se fait de manière différente surtout chez les adolescents. Jean-François Massol ${ }^{17}$ a déjà constaté cette différence de " goût» et de "dégoût" pour les œuvres lues selon le sexe du lecteur lors d'une recherche réalisée en 1997 dans quelques lycées de Grenoble.

Michèle Petit souligne aussi cette différence sous un angle psychanalytique. Selon l'auteure :

[...] à la puberté une proportion croissante de garçons - au-delà des milieux populaires - semble rejeter les livres comme s'ils s'arrachaient aux jupes de leurs mères.

I5. M. Mauss, «Les techniques du corps», dans M. Mauss, Sociologie et anthropologie, Paris, Quadrige, 2006, p. 4-II.

16. M.-C. Martin et S. Martin, Les Poésies, l'école, ouvr. cité, p. I97.

I7. J.-F. Massol, «Spontanément ou par obligation, quand les lycéens lisent des œuvres», dans Lecture privée et lecture scolaire : la question de la littérature à l'école, Grenoble, CRDP, I999, p. II5-I35. 
Ce refus se manifeste en particulier à l'égard des œuvres littéraires, dont la lecture est l'affaire des filles, ou bien de garçons qui se sont différenciés de leur entourage par leur sensibilité, leur tempérament solitaire, ou un changement consécutif à une rencontre [...]. Car la lecture, plus encore celle de textes littéraires, est en rapport avec l'expérience de la séparation, de la perte [...]. Le passage de la forme, de la lettre, vers le sens, qui suppose de faire le détour par son monde intérieur est impossible pour ceux dont l'équilibre psychique précaire repose sur son évitement ${ }^{18}$.

Pour les garçons, accepter l'idée d'un vide intérieur qui doit être rempli par la voix de l'autre, c'est-à-dire s'abandonner à un texte, se laisser emporter par la lecture littéraire, s'attacher aux mots d'un auteur ou d'un poète appartient à l'univers féminin et, selon cette logique, ils préfèrent la performance sportive comme affirmation de virilité, et laisser les qualités littéraires en particulier aux filles. C'est peut-être pour cela qu'ils préfèrent la poésie satirique, le politiquement incorrect, l'érotique, alors que les filles associent les vers au sentiment, à l'amour, aux diktats du politiquement correct. En ce sens, nous pouvons dire que la différence de sexe marque la subjectivité du lecteur.

Au Brésil, quand M. F. M. lit les sonnets d'amour de Camóes, nous remarquons immédiatement l'intérêt des filles qui soupirent à chaque vers. Quand M. S. P. apporte en classe les sonnets obscènes - et censurés dans les manuels de Bocage ${ }^{19}$ ou de Gregório de Matos $^{20}$, ce sont les garçons qui apprécient. Dans ce cas, les élèves ne reproduiraient-ils pas une construction sociale qui offre une image de la femme comme étant plus émotive, sensible, romantique, alors que l'homme affirmerait une virilité liée à l'obscène, aux provocations politiquement incorrectes? Nous pensons que c'est le cas. La subjectivité nous semble ici être plutôt une construction sociale qu'un aspect du goût personnel du lecteur.

Cette attitude provocatrice des garçons peut s'illustrer quand un lycéen de première économique et social (ES) appelé ici C., interprète à sa façon lors d'un oral de bac blanc, "Vénus anadyomène» d'Arthur Rimbaud. Selon C., Rimbaud parle, dans la première strophe, d'une "femme à cheveux bruns" qui est morte dans "un cercueil vert». Mme S. B., indignée, lui demande ce qui l'a amené à penser cela. Il ajoute alors qu' "une vieille» c'est, pour lui - il insiste sur le fait qu'il s'agit de sa propre interprétation - l'image de la mort! Tous rient, et il se justifie en expliquant que cette image «horrible» de «vieille»

I8. M. Petit, Éloge de la lecture, ouvr. cité, p. 94-95.

I9. Manuel Maria Barbosa du Bocage (I765-1805), poète portugais le plus grand représentant du néoclassicisme lusitain. Il est considéré comme l'un des plus grands auteurs de sonnets de langue portugaise, et s'est rendu célèbre pour ses poèmes obscènes, pleins de mots vulgaires. 20. Gregório de Matos (I623-I699), poète brésilien de la période baroque, surnommé «Bouche de feu " quand ses poèmes satiriques sont devenus populaires dans l'État de Bahia, sa terre natale. Comme Bocage, de Matos a écrit des poésies obscènes avec des mots grossiers. 
avec « [...] le col gras et gris, les larges omoplates / qui saillent; le dos court qui rentre et qui ressort; [...]» est l'image de la mort!

Selon C., quand le poète dit : «Puis les rondeurs des reins semblent prendre l'essor; / La graisse sous la peau paraît en feuilles plates » et, dans la troisième strophe : «L'échine est un peu rouge, et le tout sent un goût/ Horrible étrangement; on remarque surtout / Des singularités qu'il faut voir à la loupe... ", il se réfère (c'est incroyable!) «à la cellulite de cette femme». Nous pensons que les mots du troisième tercet «les reins» et «l'anus» ont mené $\mathrm{C}$. à tirer cette conclusion "créative» qui ne cache ni l'imagination ni le fantasme sexuel de l'adolescent. À ce moment-là, l'enseignante se met la main sur la tête et nous regarde, désespérée. Nous rions... C. essaie de nouveau de se justifier en disant que le poème est "sarcastique» et aussi «obscène».

Comme le montre Michèle Petit :

[...] dans l'adolescence [...] on envahit les histoires lues, avec bien sûr, le désir d'approcher les mystères du sexe. [...] Et aujourd'hui comme hier, bien des adolescents feuillettent avidement des livres parce qu'ils sont en quête de savoir sur des sujets tabous, ou pour permettre à ce qu'ils ont de plus secret de se dire, par excellence dans le domaine des rêveries érotiques, des fantasmes. [...]. Cette curiosité est la base même d'une pulsion de connaissance - la psychanalyse l'a montré - et elle pousse à chercher tous azimuts, non seulement dans les manuels d'éducation sexuelle ou les livres de médecine, mais encore dans des bandes dessinées, des témoignages, des biographies, ou de la littérature érotique $[\ldots]^{21}$

Nous ajoutons : dans les poésies également. C'est ce qu'a fait l'élève C. Pour ceux qui étaient dans la salle, l'attitude de moquerie du lycéen était évidente, c'était une stratégie pour se débarrasser du sérieux exigé par la situation d'examen blanc et également pour s'affirmer face à ses camarades. C. voulait faire rire, agir de manière provocatrice, de façon à s'imposer aux autres, à marquer son identité face au groupe. Il n'est pas compris par Mme S. B. qui a honte de la situation car, au final, selon la logique du système institutionnel, l'école n'est pas un espace pour le rire ni pour la joie (Georges Snyders) ${ }^{22}$, mais au contraire, le lieu du sérieux, du non-plaisir.

Il est alors possible de comprendre pourquoi l'interprétation de C. a un sens : c'est son univers, son fantasme (sexuel), la phase de sa vie qu'il projette sur les vers du poète symboliste. Il s'agit, selon la classification d'Annie Rouxel ${ }^{23}$, d'un «lecteur spectateur», particulièrement représentatif des lycéens de première,

2I. M. Petit, Éloge de la lecture, ouvr. cité, p. 50-51.

22. G. Snyders, Alunos felizes, ouvr. cité.

23. A. Rouxel, "Autobiographie de lecteurs et identité littéraire», dans A. Rouxel et G. Langlade, Le Sujet lecteur : lecture subjective et enseignement de la littérature, Rennes, Presses universitaires de Rennes, coll. «Didact Français», 2004, p. I46-I47. 
ou d'un «lecteur bohème», dilettante, amateur, «flâneur» qui utilise le texte comme prétexte à ses divagations. Cet élève-lecteur-spectateur-bohème (ré) invente le poème de Rimbaud sans s'inquiéter de savoir s'il est proche ou non du projet de l'auteur du texte. D'ailleurs, son interprétation peut même être le contraire. Ainsi, l'élève $\mathrm{C}$. individualise la lecture, la rend subjective, comme le justifie Serge Martin : "[...] le lecteur [devient] sujet de sa lecture parce qu’il invente le poème de sa lecture par et dans le sujet du poème ${ }^{24}$ ».

C. se comporte comme un «lecteur subjectif», selon la définition de Gérard Langlade ${ }^{25}$, un «lecteur personnel, singulier, normal» - bien différent du «lecteur expert», qu'il appelle aussi «lecteur critique, lettré, professionnel» - nous dirions «idéal» dans le sens même de l'«idéalisation» recherchée par l'école. Ainsi, toujours selon Gérard Langlade, il est un lecteur réprimé par le système scolaire :

L'exclusion, ou tout au moins la marginalisation, de la subjectivité du lecteur est couramment affichée comme une condition de réussite de la lecture littéraire scolaire et universitaire. Un exemple parmi d'autres emprunté à un document institutionnel récent ${ }^{26}:$ «Pour construire une interprétation, il faut dépasser les réactions personnelles, partielles et partiales, entachées d'erreurs, embrouillées par le jeu multiple des connotations ${ }^{27} »$.

D’après V. Jouve, il y a deux plans constitutifs de l'acte de lecture : « le plan affectif et "le plan intellectuel ${ }^{28}$ ", tous deux sont affectés par la subjectivité. Selon nous, la confusion de C. a eu lieu aussi bien sur le plan affectif que sur le plan intellectuel. Lélève a fait une lecture pleine de contresens, de façon consciente, afin de s'affirmer face à ses camarades et aux adultes en présence : la réception subjective du poème a été déviée selon les intérêts de l'adolescent.

Cependant, pour que ne perdure pas la sensation que «tout est perdu» et que se (ré)affirme la possibilité de développer une lecture subjective en milieu scolaire, émancipatrice, capable de nous libérer, de nous faire transgresser, une lecture qui nous amène à élaborer notre citoyenneté, à conquérir notre position même de sujet, notre intériorité, notre espace conscient/inconscient dans la construction d'un sujet lecteur, nous terminons cet article en rapportant ce que nous avons observé dans une classe de terminale littéraire du lycée Marie-

24. S. Martin, «Irréversible, imprévisible petite histoire d'un chroniqueur en poésie contemporaine», dans A. Rouxel et G. Langlade, Le Sujet lecteur, ouvr. cité, p. 204.

25. G. Langlade, "Le sujet lecteur auteur de la singularité de l'œuvre», dans A. Rouxel et G. Langlade, Le Sujet lecteur, ouvr. cité, p. 85.

26. Rapport de l'Inspection générale de Lettres, «La mise en œuvre du programme de français en classe de seconde", $\mathrm{n}^{\circ} 79$, oct. 2003, p. 17.

27. G. Langlade, «Le sujet lecteur auteur de la singularité de l'œuvre», dans A. Rouxel et G. Langlade, Le Sujet lecteur, ouvr. cité, p. 8I.

28. Vincent Jouve, «La lecture comme retour sur soi : de l'intérêt pédagogique des lectures subjectives", dans A. Rouxel et G. Langlade, Le Sujet lecteur, ouvr. cité, p. Io6. 
Curie à Échirolles, et qui nous fait croire, d'un commun accord avec Michèle Petit ${ }^{29}$ au rôle de la littérature - et par extension, des poésies - comme source de «lecture réparatrice».

\section{Lire Jaccottet de manière subjective au lycée}

Pour la classe de terminale littéraire, Mme V. G. a organisé la lecture de l'œuvre poétique $A$ la lumière d'hiver, de Philippe Jaccottet ${ }^{30}$ dont l'étude est exigée pour l'épreuve écrite du bac. L'ajustement de l'enseignement à l'examen national se confirme. La lecture doit d'abord se faire chez soi; toutefois, dans la dynamique de classe, Mme V. G. demande à chaque élève de lire un poème de la première série "Leçons» et, lors de chaque lecture à voix haute, la poésie est abordée de façon interprétative et analytique afin de tracer les principales thématiques travaillées par le poète dans ses textes.

Les élèves participent activement aux lectures poétiques et aux commentaires qui y sont liés. Au fur et à mesure des lectures, l'ambiance de la classe devient de plus en plus dense à cause du thème central du livre : la mort. Dans ses poésies issues de "Leçons", Jaccottet se lamente de la perte de son maître, de son disciple - sa façon de se référer à son beau-père - victime du cancer. La nature morbide, le corps douloureux, la souffrance latente dans les mots du poète cherchant à décrire la douleur de la perte angoisse toute la classe : les élèves, Mme V. G., et nous-même.

À un moment donné, une élève, visiblement émue, se lève et demande la permission de sortir. Mme V. G. lui accorde quelques minutes dehors et lui conseille de revenir quand elle se sentira mieux. Dans une ambiance tendue, les lectures poétiques continuent. Dix minutes passent et une autre élève demande à sortir pour aller voir son amie qui ne revient pas. L'enseignante la remercie de sa gentillesse. Les deux lycéennes restent dehors pendant presque une demi-heure et ne rentrent qu'à la fin du cours. Par la suite, l'enseignante nous explique que le père de la première élève est malade du cancer et que la mère de la deuxième est en phase terminale de cette maladie.

Voilà la poésie, dans son exercice éthique et esthétique ${ }^{31}$, capable de toucher le corps et, par extension, l'âme, dirait Jean-Luc Nancy ${ }^{32}$. Vraiment? Est-il

29. M. Petit, Éloge de la lecture, ouvr. cité, p. 99.

30. P. Jaccottet, $A$ la lumière d'hiver [1977], précédé de Leçons et de Chants d'en bas [1983] et suivi de Pensées sous les nuages [1994], Paris, Gallimard, 1994.

3I. C. A. de B. Neves, Poesia na sala de aula: um exercício ético e estético, master en Éducation, Pontifícia Universidade Católica de Campinas, 2008, 190 p.

32. J.-L. Nancy, Corpus, Paris, Éditions Métailié, 2006. 
possible d'affirmer que les textes poétiques de Jaccottet aient pu toucher les sentiments de ces deux élèves, réveillant leur douleur? Ou, submergées par leur douleur, ne se seraient-elles pas laissé dépasser en lisant le poète? Impossible à dire.

Ou encore, dans une autre perspective peut-être plus cruelle, ne serait-ce pas l'école qui les a exclues, puisque les adolescentes se sont senties obligées de se retirer de la classe pour ne pas perturber les autres avec leur douleur? Si l'institution scolaire n'est pas l'espace du rire, de la joie, comme nous le lui avons reproché précédemment, elle n'est pas non plus celui des pleurs, de la douleur, de la mort. Ce climat donne l'impression qu'il faut se contenir tout le temps, car le fait de se laisser aller peut ne pas être bien perçu.

Si elles ne trouvent pas d'appui dans le milieu scolaire, les élèves peuvent chercher à se consoler à travers les lectures. Nous pensons que, d'une certaine façon, la lecture poétique de Jaccottet pourrait fonctionner pour elles comme une lecture réparatrice, accueillante, autothérapeutique - comme la qualifie bien Michèle Petit - celle qui nous sauve en temps de crise :

Mais tout au long de la vie, dans les moments de crise, la lecture peut être une voie privilégiée pour nous faire retrouver un espace paisible et l'expérience de l'enfant qui, à partir de cet espace esthétique, calme, protecteur, entre sa mère et lui, se ressource et devient autonome. Si elle permet de se découvrir ou de se construire, elle devient cruciale lorsque l'on doit se reconstruire, après un deuil, une maladie, un accident, une séparation amoureuse, la perte de son emploi, une dépression, toutes épreuves dont nos destins sont faits, toutes situations qui mettent à mal la représentation que l'on a de soi et le sens de sa vie. La lecture joue alors le rôle d'autothérapie ${ }^{33}$.

La lecture des poésies de Jaccottet peut permettre aux élèves d'élaborer ou de restaurer leur espace intime, leur individualité en les aidant à résister à la fragilité physique et psychique dans laquelle ils se trouvent. Par la lecture littéraire, ils peuvent (re)conquérir leur position de sujet, leur dignité, leur condition humaine, effectuer un travail quasi psychanalytique de dépassement de la douleur et, ainsi, de (re)construction de soi. Non pas que la lecture poétique puisse tout réparer, dans leur cas. Il serait naïf de le croire, reconnait l'auteure, «mais parfois [elle peut] contribuer à symboliser ses pulsions destructrices, à élaborer sa pensée, à donner une plus grande liberté pour se porter ailleurs que sur les chemins tout tracés par le destin ${ }^{34}$ ».

La réception de la lecture de Jaccottet dans cette classe de terminale littéraire devrait sensibiliser tout le monde, c'est un «événement poétique» selon

33. M. Petit, Éloge de la lecture, ouvr. cité, p. Ioo.

34. Ibid., p. Iо. 
l'expression de Nathalie Rannou. Nous partageons l'idée que l'école doit promouvoir ce type de lecture subjective "qui [concourt] au développement de l'élève en tant que personne, [laisse] une place entière à l'expression et la réalité du sujet. L'élève est un lecteur global, aussi complexe, paradoxal, incarné, contextualisé, clivé, en devenir... que n'importe quel lecteur expert ${ }^{35}$ ».

Reprenons ainsi le titre de cet article et reconnaissons que promouvoir cette lecture littéraire, poétique, subjective entre les lycéens est un défi pour les enseignants. Toutefois, c'est un défi possible aussi bien au Brésil qu'en France, surtout à l'heure actuelle où les élèves exigent de nous, professeurs, une disposition à négocier les savoirs allant au-delà du savoir que l'on doit conserver, mais aussi amenant à un savoir-faire et, pourquoi pas, à une poïétique. Si l'on considère toujours l'épigraphe qui ouvre ce travail, à travers les poèmes de Jaccottet les élèves de terminale auxquelles nous faisons référence peuvent (se) lire, (se) dire, (s')écrire chacune dans sa douleur.

35. N. Brillant Rannou, Le lecteur et son poème. Lire en poésie : expérience littéraire et enjeux pour l'enseignement du français en lycée, thèse de doctorat en littérature française, université de Rennes 2, 20IO, 649 p. 